\title{
The late stages of pulmonary sarcoidosis
}

\author{
J. G. SCADDING
}

The Brompton Hospital, London, S.W.3

\begin{abstract}
Summary
Pulmonary sarcoidosis is frequently preceded by bilateral hilar lymph-node enlargement (BHL). Although it is normally difficult to be certain in a patient without BHL that a stage of BHL has not in fact occurred in the past but been missed, there is good evidence that some cases of pulmonary sarcoidosis arise without preceding BHL. Although a prefibrotic infiltration of the lungs will usually show signs either of resolution or of developing fibrosis within 2 years, such an infiltration may remain apparently nonprogressive, producing little functional defect, for much longer than this, and in such a case, substantial resolution remains possible. A high proportion of those who develop fibrosis show a characteristic pattern of strand-like fibrosis in the middle zones and lower part of the upper zones of the lungs, with emphysematous changes above and below. In densely fibrotic parts of the lungs, cavities may appear, probably from necrosis at the centres of large masses of hyaline fibrosis. A rare complication is the development of a 'fungus ball' due to the growth of Aspergillus in such a cavity.

Occasionally, fine focal fibrosis develops without distortion of lung architecture, but with severe disability.
\end{abstract}

A rare but important event is the development of bronchial stenoses. These may affect main, segmental or subsegmental bronchi, more especially the proximal parts of segmental or subsegmental bronchi.

Calcification has been observed to develop in a characteristic symmetrical fashion in the hilar lymphnodes and occasionally in small foci in the lungs in about $5 \%$ of patients with pulmonary sarcoidosis who initially had BHL, followed for periods ranging from 5 to 20 years.

In the late stages of sarcoid fibrosis of the lungs, the specific granuloma may be no longer convincingly demonstrable.

It will be helpful, before discussing the late stages of pulmonary sarcoidosis, to survey briefly the commoner early patterns of mediastinal lymph-node and pulmonary sarcoidosis. These are so well recognized that radiographic record of a charac- teristic sequence of changes may be sufficient to establish diagnosis with little risk of error.

Bilateral hilar lymph-node enlargement (BHL), sometimes evidently involving also the right paratracheal group, is often the first evidence of the disease. It may be discovered in persons with few or no symptoms as a result of routine radiography, or may be associated with erythema nodosum, febrile arthralgia often involving particularly the ankles, or constitutional symptoms with low fever. This initial illness, if there is one, is generally selfterminating. In the majority of cases, the BHL subsides within 6 months to 2 years. In some cases, about two-fifths in my experience, BHL is followed by a widespread pulmonary infiltration causing generalized mottled shadowing in the chest radiograph. In a high proportion of cases, this prefibrotic infiltration in its turn resolves either completely or leaving minor presumably fibrotic residues. Among patients who presented with BHL and no radiographic changes in the lungs when first seen, I found that $8 \%$ of those without symptoms or with erythema nodosum showed some lung fibrosis 2 years later, but only one-third of these, about one in forty of the total group, had respiratory symptoms. A higher proportion, one in four, of the small number who at the onset had respiratory or constitutional symptoms, without erythema nodosum, were left with some residual fibrosis, though in most instances this produced only slight or no symptoms.

The time-scale of the sequence BHL-infiltrationresolution with or without some residual fibrosis is very variable. The whole process may be completed to resolution within one year; most frequently it takes 2-3 years; but occasionally it is much slower, as in the case illustrated in Figs. 1-4.

Many patients show pulmonary infiltration as the first observed evidence of sarcoidosis. In my experience about equal numbers of those presenting in this way have and have not evidence of past or present BHL. It is only occasionally possible to be sure that a patient presenting without BHL has not passed through a phase of BHL; but it is significant that patients in whom there is no record of BHL 


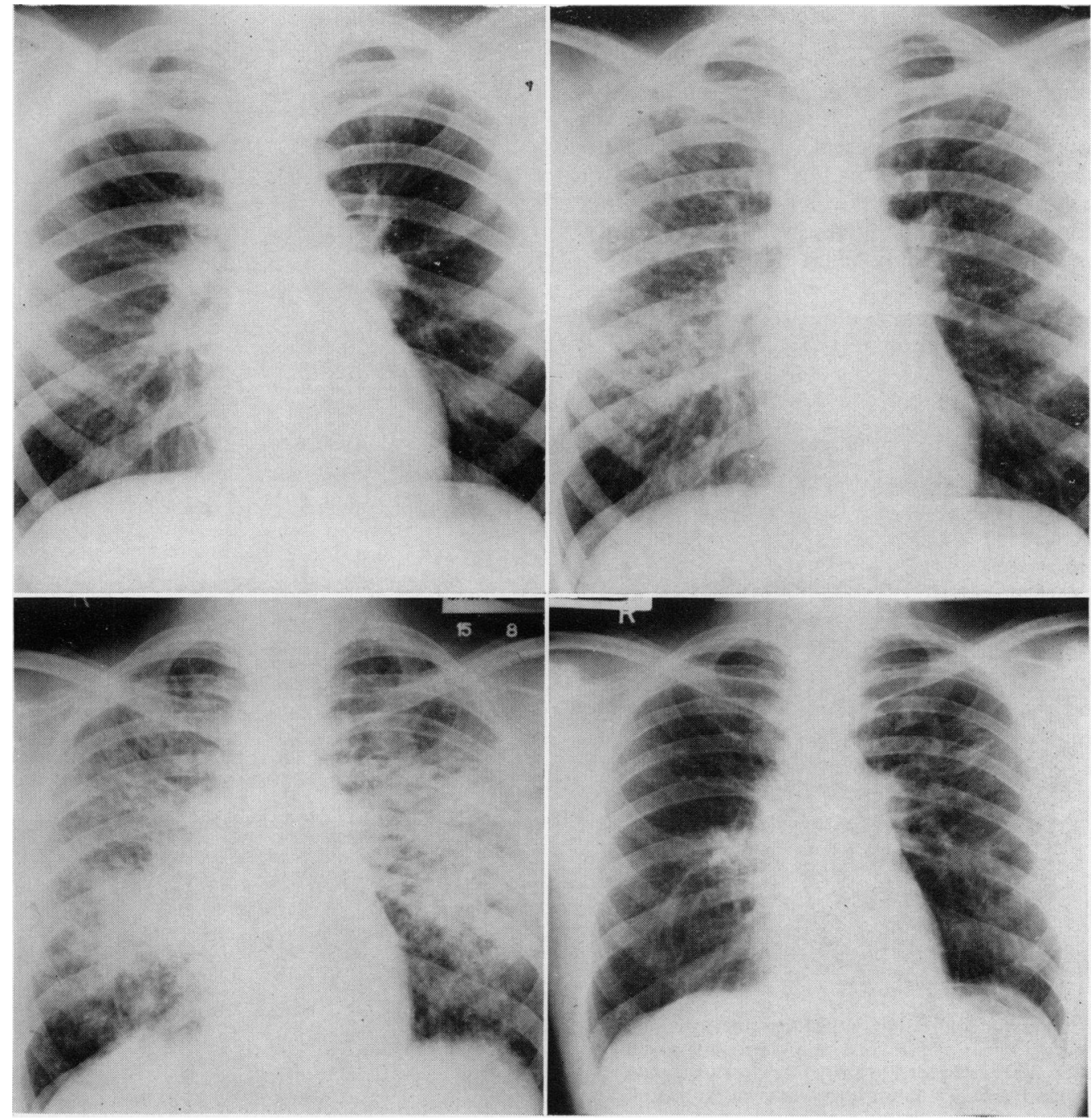

FIG. 1 (top left). BHL in a symptomless man, aged 19, in March 1957. It was known to have been present for nearly 3 years. The Kveim test was positive.

Fig. 3 (bottom left). The same, August 1963. Still symptom-free. Objective tests showed only a slight restrictive ventilatory defect, and $\mathrm{CO}$ transfer factor in the normal range $(26.5 \mathrm{ml} / \mathrm{min} / \mathrm{mmHg}$ by the steady state method).

differ from those with evidence of BHL in having a lower incidence of evident extrathoracic sarcoidosis and a rather higher incidence of subsequent fibrosis.

Of eighty-seven patients with prefibrotic sarcoidosis of the lungs, with or without BHL, I found that twenty-five had evidence of some permanent fibrosis after 5 years' observation, though only a minority of these had appreciable disability (Scad-

FIG. 2 (top right). The same, May 1959. Still symptomfree, playing Rugby football. Maximum voluntary ventilation $172 \mathrm{l} / \mathrm{min}$.

Fig. 4 (bottom right). The same, December 1967. The dense infiltration shown in Fig. 3 has resolved without 'treatment' at any time, leaving radiological evidence of slight fibrosis in the left middle zone. CO transfer factor $27.5 \mathrm{ml} / \mathrm{min} / \mathrm{mmHg}$.

ding, 1967). Thus the proportion of patients with intrathoracic sarcoidosis in England who develop disabling fibrosis is small. It is necessary to specify the place where the observations were made, since the prognosis of sarcoidosis varies in different populations; in particular, north American Negroes tend to have more extensive and florid disease than those of Caucasian stock and a correspondingly worse prognosis. 
In a few patients, a prefibrotic infiltration remains non-progressive for prolonged periods. If such a patient has no symptoms, substantial resolution with only minor radiographic residues and no disability can still occur, no matter how long the infiltration has been present. For instance, the irregular densities shown in Fig. 5 were present for 5 years in a woman in the late twenties, without symptoms, but eventually resolved spontaneously, leaving almost clear lung-fields.

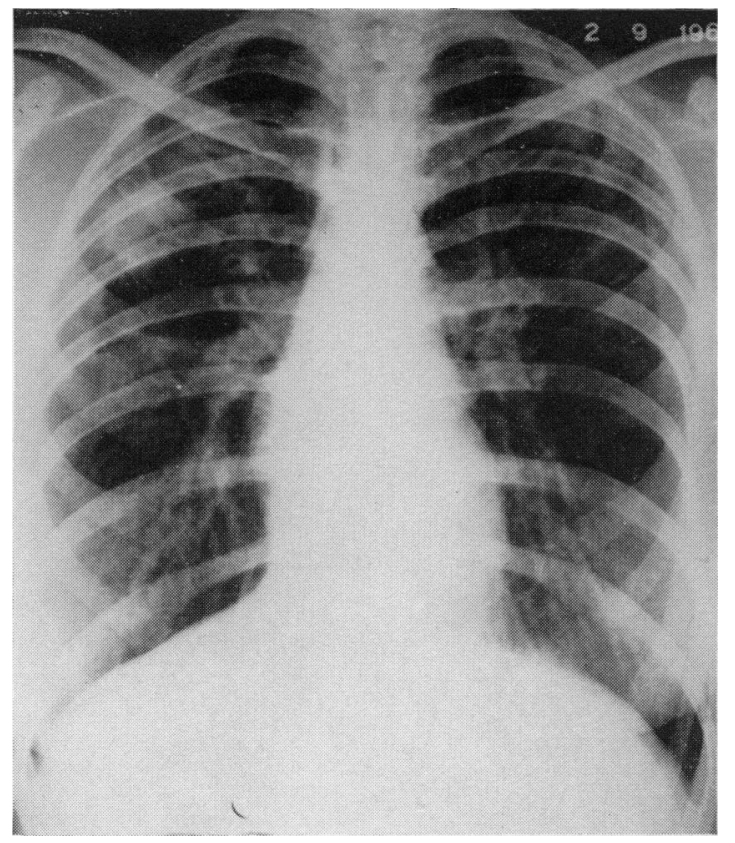

FIG. 5. Sarcoid infiltration of the lungs, locally confluent in the right upper/middle zone, in a symptomless woman aged 27. It persisted for 5 years before clearing spontaneously, leaving only a minor linear 'scar' at the site of the confluent infiltration.

A high proportion of those developing fibrosis show a rather characteristic radiographic appearance. In the earlier stage, mottled opacities are densest in the middle or the middle and adjacent parts of the upper zones of the lungs, the lower and, usually less strikingly, the upper zones being clearer. Irregular linear shadows appear to extend out from the hila (Fig. 6). When fibrosis is established, the hilar shadows are often raised, with corresponding distortion of the vascular pattern; mottling tends to disappear, the linear shadows already mentioned become more prominent, and there may be localized dense shadows, presumably of confluent fibrosis (Fig. 7). The lower and sometimes the upper zones of the lungs show areas of transradiancy suggesting emphysema, sometimes bullous; and air-containing

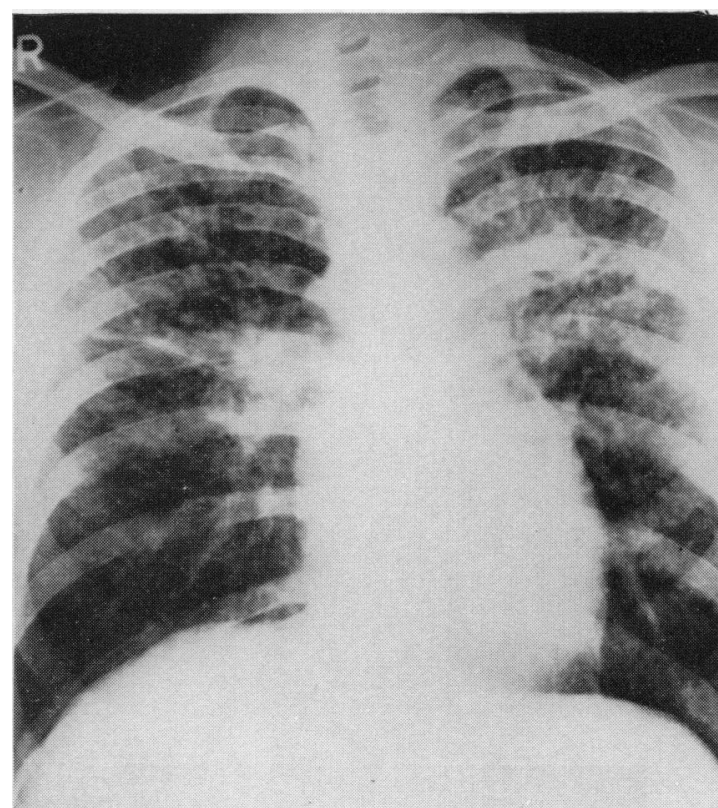

Fig. 6. A characteristic pattern of developing middlezone fibrosis in a man. Widespread mottling had first been observed 18 months earlier. Note the concentration of mottling in the middle zone and the adjacent part of the upper zone, and the tendency to a linear pattern of shadowing from the hila into these densely involved areas.

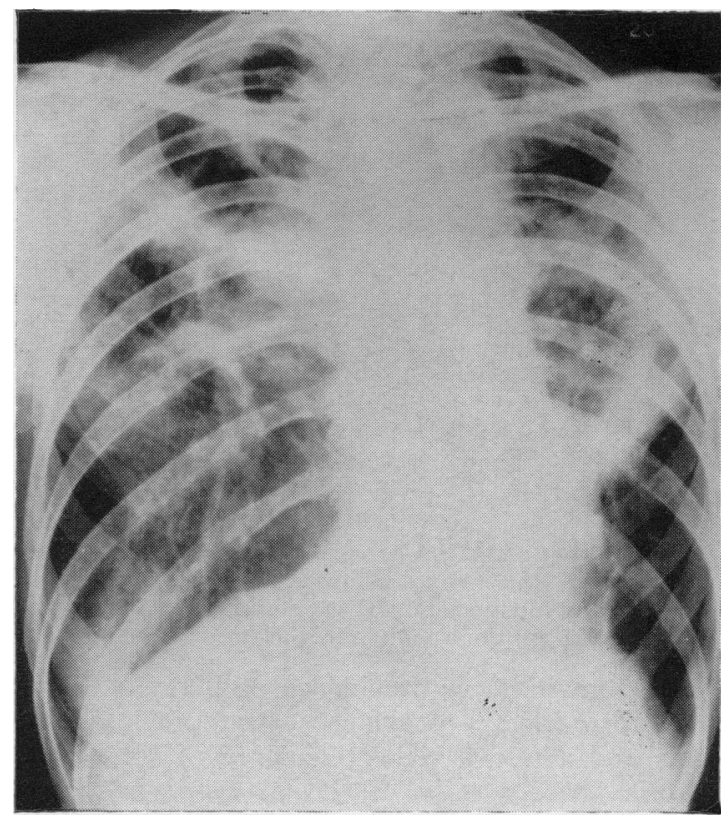

FIG. 7. Confluent shadows, principally in the middle zones of the lungs in a woman aged 39 who had had BHL 10 years earlier, and whose disease had progressed to the stage shown through a widespread infiltration of the lungs. 
spaces may be evident, especially on tomography, in in the densely opaque areas. These have been shown in some instances at necropsy to have dense fibrous tissue walls, without specific granulomatous or other changes or epithelial lining (Figs. 8 and 9).

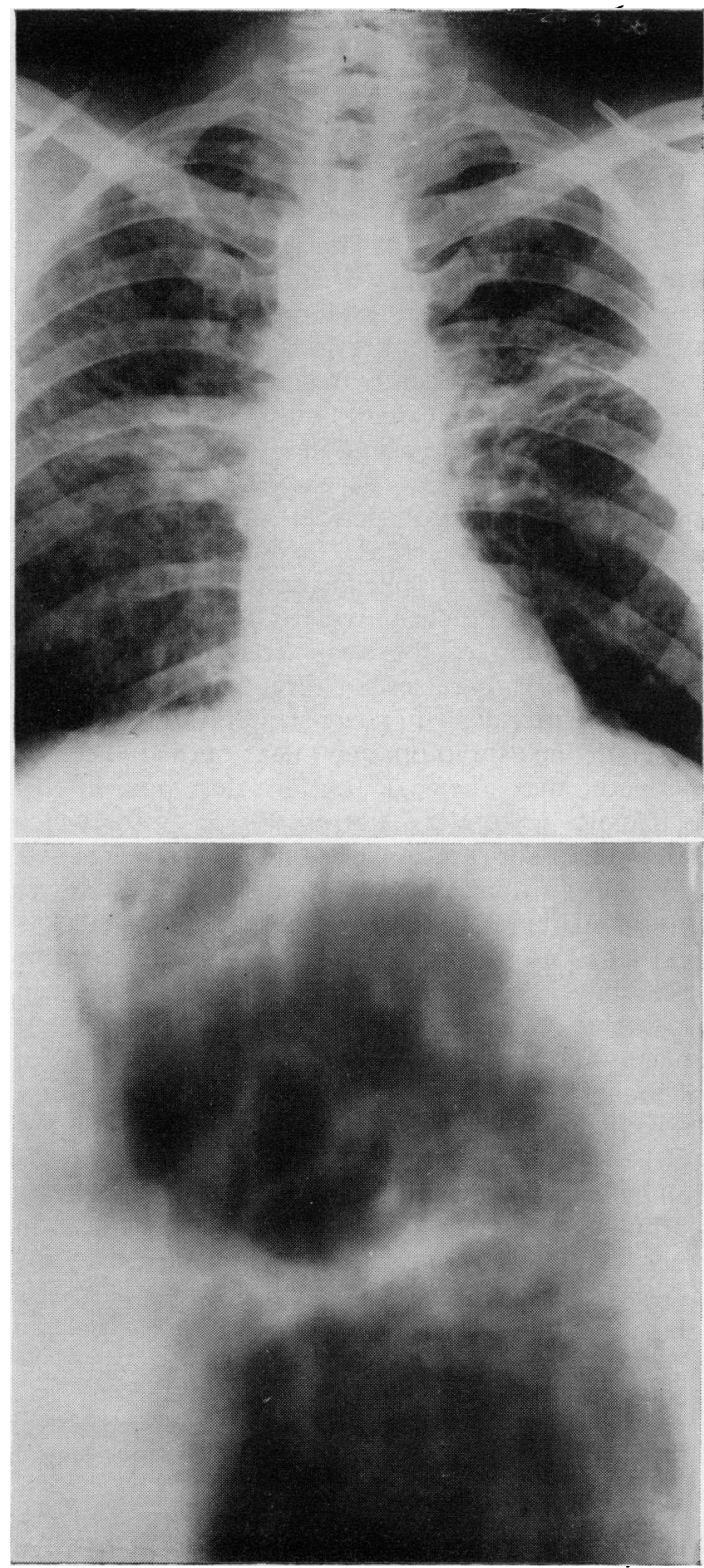

FIG. 8 (top). Fibrotic stage of pulmonary sarcoidosis in a man aged 42 , in whom a widespread fine mottled infiltration had first been observed 9 years earlier.

Fig. 9 (bottom). Tomogram of middle zone of the left lung, $12 \mathrm{~cm}$ from the back, in the same patient, showing irregular cavities in confluent fibrosis.
Occasionally, these air-containing spaces, like those of other origin, become colonized by the common mould Aspergillus, usually the species fumigatus. This results in the development of a 'fungus ball', presenting a characteristic radiographic appearance (Figs. 10 and 11).

The usual pattern of functional defect consists in a restrictive ventilatory defect (small total lung capacity with proportionate reduction in its subdivisions, possibly diminished compliance, and normal airways resistance) and a gas transfer defect (reduced transfer factor, tendency to arterial oxygen desaturation at rest and increasing on exercise, with normal or slightly low $\mathrm{CO}_{2}$ pressure). At the stage of prefibrotic infiltration, the results of all these tests may be within normal limits, in spite of a gross radiographic abnormality; if they are abnormal, ventilatory restriction is generally more prominent than gas transfer defect. With the development of fibrosis, the gas transfer defect becomes more prominent. Chronic bronchitis is so frequent in a cigarette-smoking population that it is not surprising that a proportion of patients show an additional obstructive ventilatory defect from this cause.

Not all those patients developing fibrosis show the 'middle zone' pattern described above. In a few, a widespread granulomatous infiltration appears to undergo focal fibrosis, without confluence or evident distortion of macroscopic lung architecture. In these, the mottling becomes less dense in the radiograph, individual elements diminishing in size, and perhaps giving place to a fine reticular pattern; but disability increases, and objective tests show deterioration in transfer factor and in restrictive ventilatory defect. An even rarer development is airways obstruction with grossly overdistended lungs, suggestive of emphysema. I have seen only one example of this. The patient was a woman who was a non-smoker and had no evidence of chronic bronchitis at any time. At the onset, she showed radiographic evidence of BHL with widespread infiltration (Fig. 12); over the next 2 years she became increasingly breathless on exertion, with increased density of the mottling in the lung fields. At this time the FEV 1 was only $800 \mathrm{ml}$, constituting $47 \%$ of the FVC, and the gas transfer factor was only moderately lowered, to about two-thirds of the predicted value. In view of her symptoms, she was treated with corticotrophin, with symptomatic and radiographic improvement, but persistence of airways obstruction. The infiltration reappeared on cessation of the corticotrophin treatment after 18 months, but subsequently disappeared spontaneously, leaving persistent airways obstruction, very low gas transfer factor not increasing on exercise, and radiographic evidence of overdistended abnormally transradiant lungs (Fig. 13). 


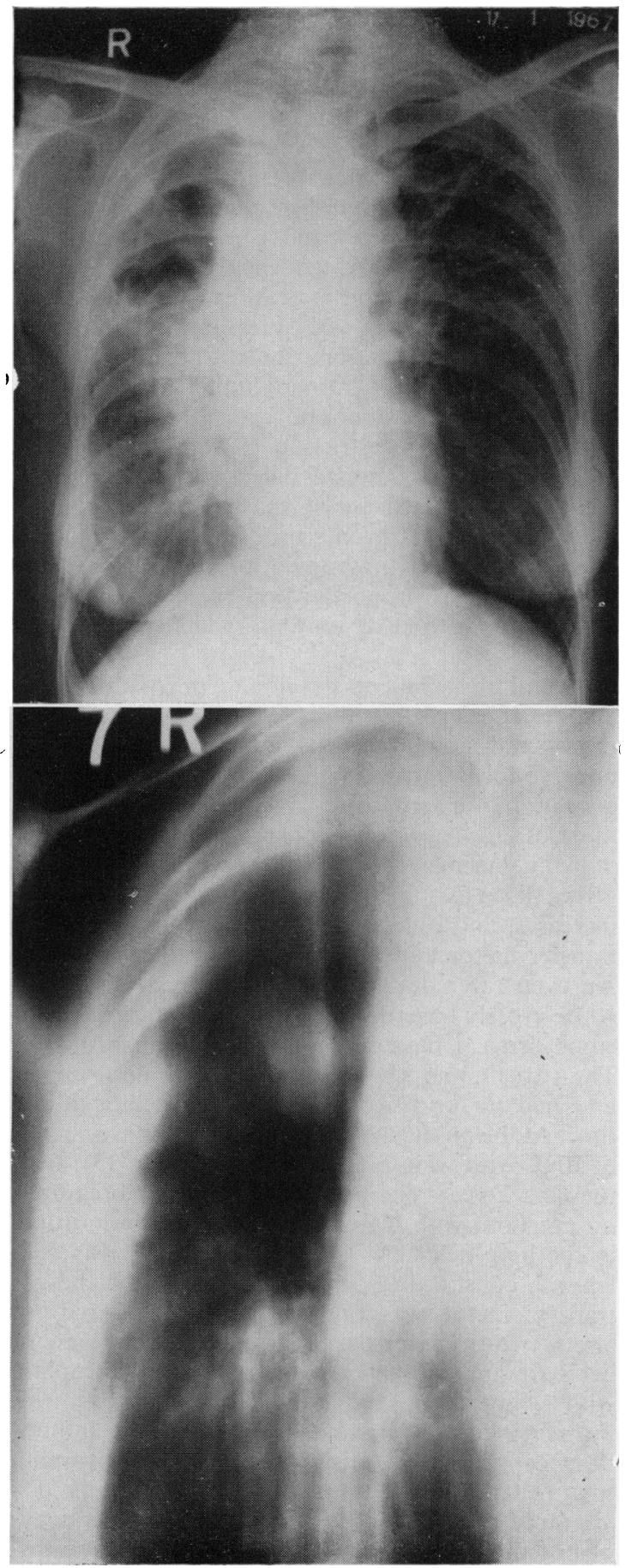

Involvement of main, lobar, segmental and large sub-segmental bronchi in sarcoidosis is well recognized. This occasionally gives rise in the later stage to bronchial narrowings; these have a rather characteristic distribution affecting the proximal parts of segmental or subsegmental bronchi, though occasionally main bronchi may show long smooth narrowings (Fig. 14). Clinically, this produces dyspnoea with a pattern of inspiratory as well as expiratory obstruction, causing prolongation and noisiness of both inspiration and expiration (Citron \& Scadding, 1957).

Another feature of the late stage of intrathoracic sarcoidosis the frequency of which has only recently been recognized is the development of calcification, typically in symmetrical fashion in the hilar lymphnodes, and less frequently in small foci in the lungs. In the hilar nodes, it occurs only, in my experience, in patients who have also had a pulmonary infiltration; I found that it developed in $5 \%$ of such patients, observed for periods ranging from 5 to 20 years (Scadding, 1961, 1968a). The pattern of discrete symmetrical calcifications in the hilar and sometimes paratracheal regions, corresponding to the distribution of the early enlargement of hilar nodes, is very characteristic (Fig. 15). Occasionally, the nodes are calcified in 'egg-shell' fashion, showing a well-defined radio-opaque linear outline. There is evidence that the calcification develops in the ischaemic featureless fibrous tissue into which sarcoid tubercles are converted if they do not resolve.

Finally, it must be noted that in the latest stages of sarcoid fibrosis of the lungs, the specific granuloma may be no longer convincingly demonstrable (Scadding, 1968b). In such cases, there is much featureless hyaline fibrosis in lymph-nodes, lungs, spleen and sometimes other organs; groups of Schaumann conchoidal bodies may be found in this, though careful search may be required; and the few epithelioid cells that remain no longer show a follicular arrangement. In such cases, although the pathologist may remain unconvinced, the physician may legitimately conclude, from knowledge of the clinical course and earlier history of the case, that a diagnosis of sarcoidosis is justified, since he can

Fig. 10 (top). Fibrotic stage of pulmonary sarcoidosis in a woman aged 59: duration unknown, since she came under observation with established fibrosis. In the upper half of the right lung, there is a thick-walled cavity. She also had a plaque-like sarcoid of the skin of the right forearm, and sarcoid infiltration of some old scars.

FIG. 11 (bottom). Tomogram of the upper half of the right lung in the same patient: the well-defined opacity in the cavity was shown to be an aspergilloma by the finding of $A$. fumigatus in the sputum, and the presence of precipitins, giving multiple strong lines in gel diffusion tests, in the blood serum. 


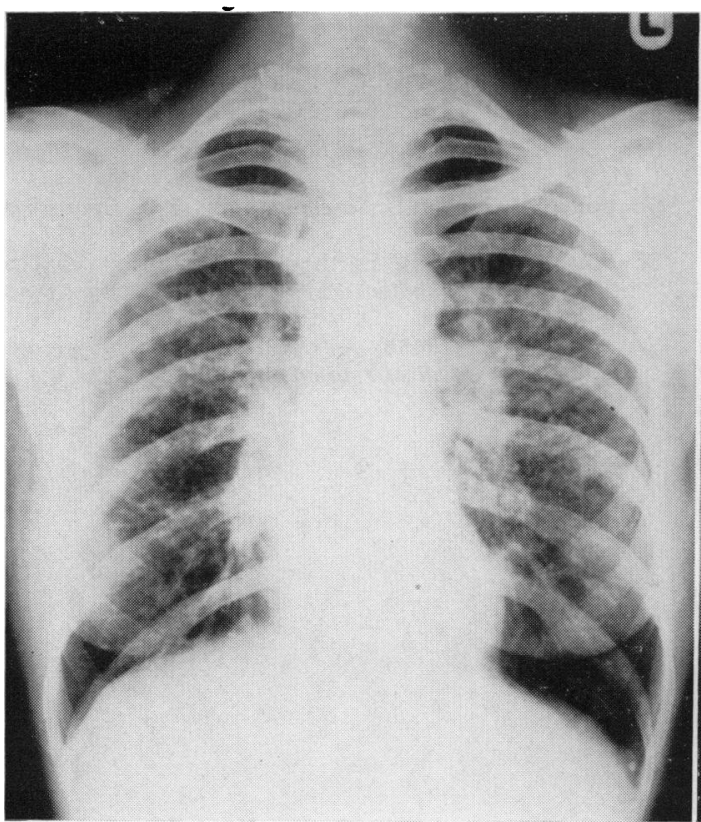

FIG. 12. BHL and widespread infiltration of the lungs in a woman aged 40: see text.

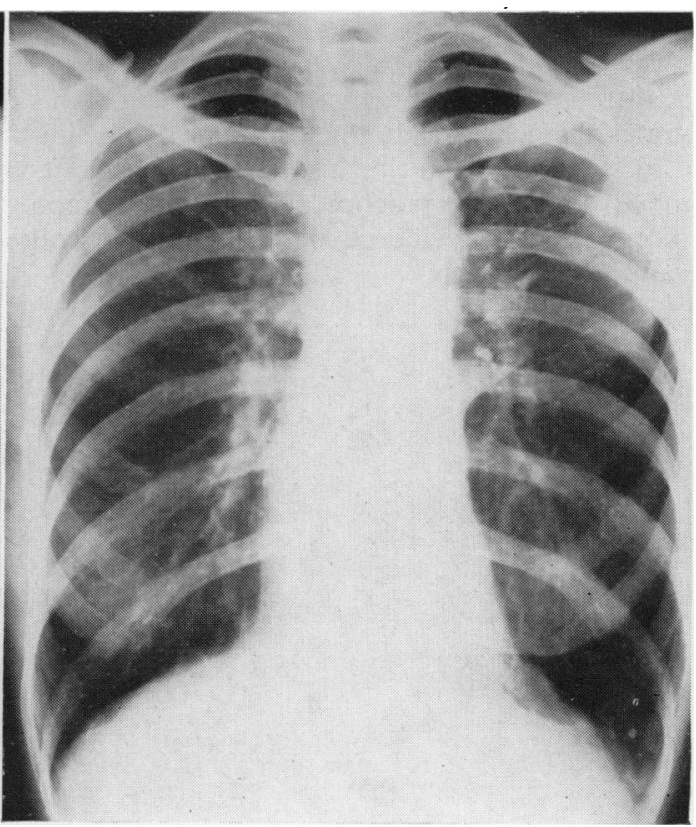

Fig. 13. The same, 11 years later. The lungs appear over-distended; there was severe airways obstruction and low gas-transfer factor.

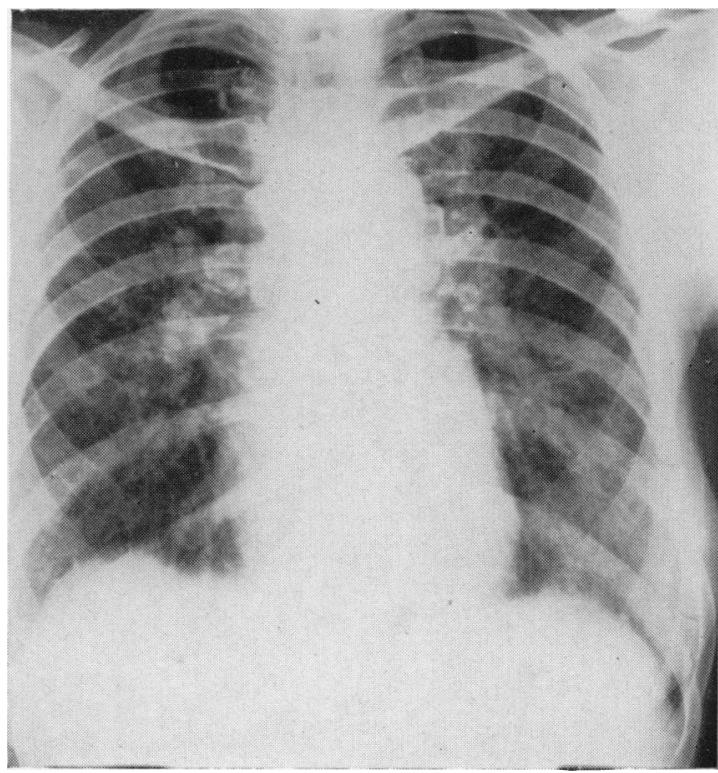

Fig. 15. Symmetrical calcification in hilar lymph-nodes. Sarcoid BHL had been discovered 13 years earlier and had been followed by widespread infiltration in the lungs.
FIG. 14. Bronchogram showing narrowing of main bronchi by fibrosing sarcoid infiltration in a man aged 38 years. 
deduce that in the active stage the defining characteristics of sarcoidosis, non-caseating epithelioid-cell tubercules in several organs, were present. This illustrates the general rule that although the definition of sarcoidosis is in histological terms, its diagnosis depends in practice almost always upon the correlation of evidence derived from several relevant fields of study.

\section{References}

Citron, K.M. \& Scadding, J.G. (1957) Stenosing noncaseating tuberculosis (sarcoidosis) of the bronchi. Thorax, $12,10$.

SCADDING, J.G. (1961) Calcification in sarcoidosis. Tubercle, 42, 121.

Scadding, J.G. (1967) Sarcoidosis. Eyre \& Spottiswoode, London.

SCADDING, J.G. (1968a) Further observations on calcification in sarcoidosis. Scandinavian Journal of Respiratory Diseases, suppl. 65, 235.

SCADDING, J.G. (1968b) A 'burnt-out' case of sarcoidosis. Postgraduate Medical Journal, 44, 105. 